\title{
ON THE COMPACTIFICATIONS OF ARITHMETIC QUOTIENTS OF SYMMETRIC SPACES
}

\author{
BY PETER KIERNAN
}

Communicated by S. S. Chern, July 6, 1973

The purpose of this short note is to combine the results in [3] and [4] to prove the following:

TheOREM. Let $X$ be a bounded symmetric domain and let $\Gamma$ be an arithmetically defined group of automorphisms of $X$. Let $V^{*}$ be the Baily-BorelSatake compactification of $V=X / \Gamma$ and let $V^{* *}$ be the set $V^{*}$ with the topology defined by Piateckii-Šapiro in [5]. The identity mapping $i: V^{*} \rightarrow V^{* *}$ is a homeomorphism.

Proof. We shall use the notation in [3] and [4]. By [1], the identity mapping $i: V^{*} \rightarrow V^{* *}$ is continuous. Since $V^{*}$ is compact and Hausdorff, $i$ is a homeomorphism if and only if $V^{* *}$ is Hausdorff. Let $j: V \rightarrow V$ be the identity. By Theorem B of [3], $V$ is hyperbolically imbedded in $V^{*}$. The proof of Theorem 1 in [4] shows that $j$ extends to a continuous map $j: V^{* *} \rightarrow V^{*}$. It is important to note that the proof does not make use of the fact that $V^{* *}$ is Hausdorff. Clearly $j$ is a continuous inverse to $i$ since $V$ is dense in $V^{*}$. Q.E.D.

Armand Borel has proven the previous theorem by a more general and more natural method. He can show that the topology of Satake compactifications can be defined by means of analogues of cylindrical sets and therefore without using fundamental sets. However, since it will be a while before he publishes his result, the theorem above does clarify the question concerning the equivalence of the compactifications $V^{*}$ and $V^{* *}$.

\section{REFERENCES}

1. W. L. Baily, Jr., Fourier-Jacobi series, Algebraic groups and Discontinuous Subgroups, Proc. Sympos. Pure Math., vol. 9, Amer. Math. Soc., Providence, R.I., 1966, pp. 296-300. MR 36 \#2834.

2. W. L. Baily, Jr., and A. Borel, Compactification of arithmetic quotients of bounded symmetric domains, Ann. of Math. (2) 84 (1966), 442-528. MR 35 \#6870.

3. A. Borel, Some metric properties of arithmetic quotients of symmetric spaces, J. Differential Geometry 6 (1972), 543-560.

AMS (MOS) subject classifications (1970). Primary 32M15. 
4. P. Kiernan and S. Kobayashi, Satake compactification and extension of holomorphic mappings, Invent. Math. 16 (1972), 237-248.

5. I. I. Pjateckii-Šapiro, Arithmetic groups in complex domains, Uspehi Mat. Nauk 19 (1964), no. 6 (120), 93-121=Russian Math. Surveys 19 (1964), no. 6, 83-109. MR 32 \#7790.

Department of Mathematics, University of British Columbia, Vancouver 8, British Columbia, Canada

Current address: Mathematisches Institut, Universitat Bonn, 53 Bonn, Germany 